- EDTTORIAL • PULSANDO LA CUESTIÓN SOBRE LA VIVIENDA Y EL HÁBITAT / PRESSING THE HOUSING AND HABTTAT QUESTION, Rosa María Añón Abajas • ENTRE LínEAS • THE PRIMACY OF RESIDENTIAL QUALITY IN URBAN CREATION. A CURRENT OBSERVATION ON A RECURRING NOTION / LA PRIMACÍA DE LA CALIDAD RESIDENCIAL EN LA CREACIÓN URBANA UNA OBSERVACIÓN ACTUAL DE UNA NOCIÓN RECURRENTE. Sten Gromarko • ARTÍCULOS • HACIA LA NUEVA SOCIEDAD COMUNISTA: LA CASA DE TRANSICIÓN DEL NARKOMFIN, EPILOGO DE UNA INVESTIGACIÓN / TOWARDS THE NEW COMMUNIST SOCIETY: THE TRANSITION HOUSE OF NARKOMFIN, A RESEARCH EPILOGUE. Daniel Movilla Vega; Carmen Espegel AlonSO • EL SISTEMA COMO LUGAR. TRES ESTRATEGIAS DE COLECTIVIZACIÓN DEL ESPACIO DOMÉSTICO CONTEMPORÁNEO I THE SYSTEM AS A PLACE. THREE COLLECTIVIZATION STRATEGIES OF THE CONTEMPORARY DOMESTIC SPACE. Fernando Nieto Fernández • LA CASA EN "CAMPO DE ARROZ". UN IDEOGRAMA DE INTERACCIÓN EN EL HÁBITAT JAPONÉS CONTEMPORÁNEO / HOUSE IN A "RICE PADDY". AN IDEOGRAM OF INTERACTION IN THE CONTEMPORARY JAPANESE HABITAT. Pedro Luis Gallego Fernández • LA CONSTRUCCIÓN TEORICA Y PRÁCTICA DE UN NUEVO HÁBTTAT MODERNO: UNOS PATIOS Y UNA CALLE (1946-1954) THE THEORETICAL AND PRACTICAL CONSTRUCTION OF A NEW MODERN HABITAT: SOME PATIOS AND A STREET (1946-1954). Juan Pedro Sanz Alarcón, Miguel Centellas Soler, Pedro García Martínez • EL BARRIO DE SÃo VICTOR DE ÁLVARO SIZA: ENTRE LA TEORÍA Y LA PRÁCTICA DE LAS OPERACIONES SAAL / DISTRICT OF SÃO VICTOR OF ÁLVARO SIZA: BETWEEN THEORY AND PRACTIC OF THE SAAL OPERATIONS. Aitor Varea OrO • REVISITING PUBLIC SPACE IN POST-WAR SOCIAL HOUSING IN GREAT BRITAIN REPENSANDO EL ESPACIO PÚBLICO DE LAS VIVIENDAS SOCIALES DE POST-GUERRA EN GRAN BRETAÑA. Pablo Sendra • INTIMIDADES TRANSGREDIDAS: HABITAR EN TRÁNSITO / TRANSGRESSED PRIVACIES: TO LIVE IN TRANSIT. Maŕa Prieto Peinado • RESEÑAS BIBLIOGRÁFICAS • R.D. MARTIENSSEN: LA IDEA DEL ESPACIO EN LA ARQUITECTURA GRIEGA. Juan Luis Trillo de Leyva

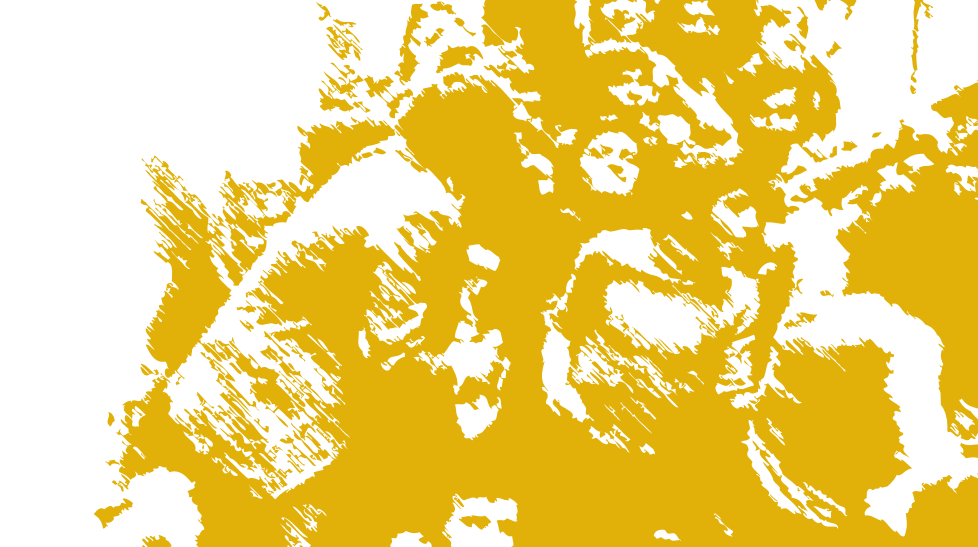

(n)
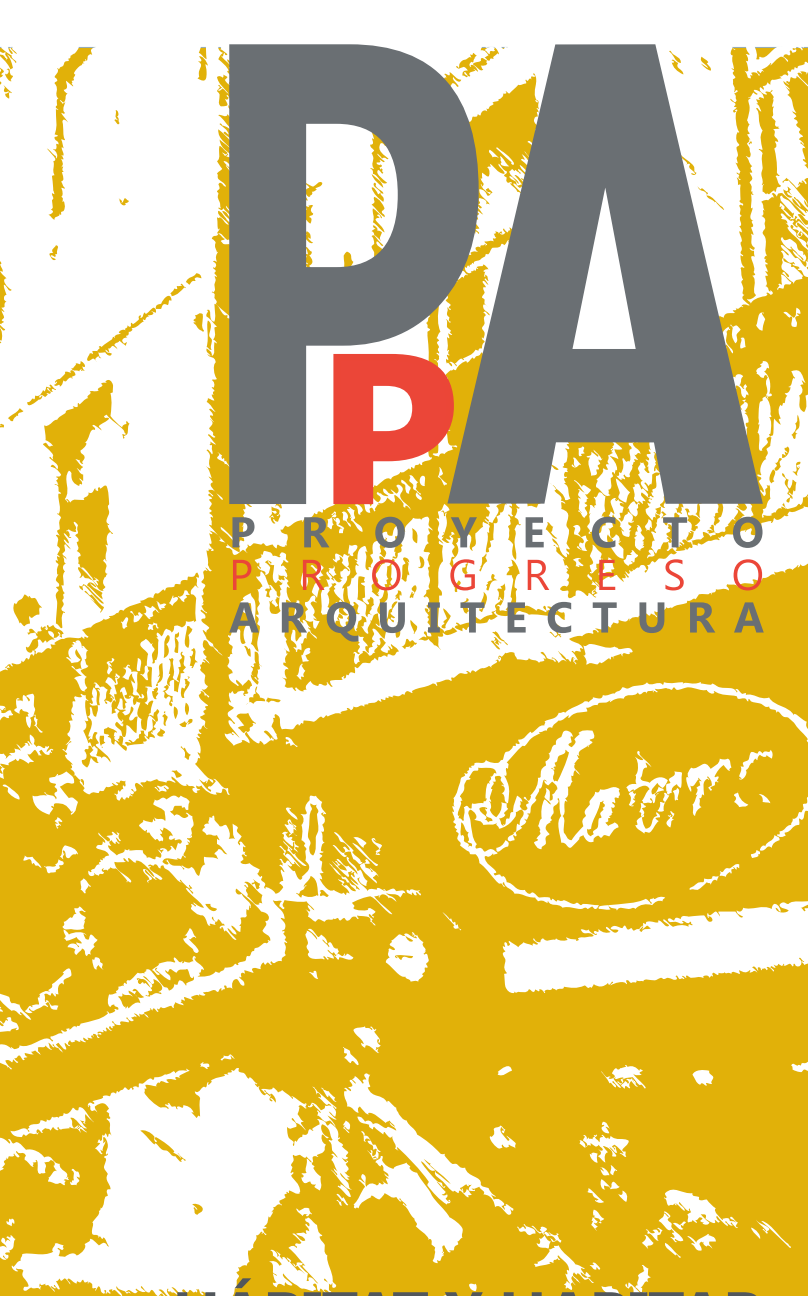

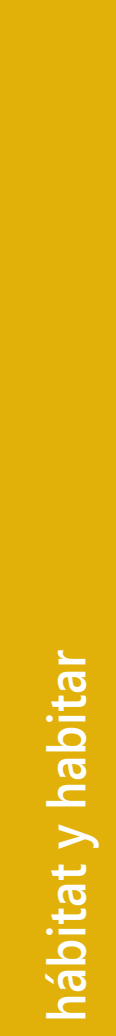

N9 


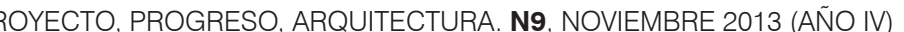

hábitat y habitar

editorial
PULSANDO LA CUESTIÓN SOBRE LA VIVIENDA Y EL HÁBITAT / PRESSING THE HOUSING AND HABBTAT QUEESTION
Rosa María Añón Abajas

entre líneas

THE PRIMACY OF RESIDENTIAL QUALITY IN URBAN CREATION. A CURRENT OBSERVATION ON A RECU UNAen GrOBSERVACION ACTUAL DE UNA NOCIÓN RECURRENTE

artículos

HACIA LA NUEVA SOCIEDAD COMUNISTA: LA CASA DE TRANSICIÓN DEL NARKOMFIN, HOUSE OF NARKOMFIN A RESEARCH EPILOGUE

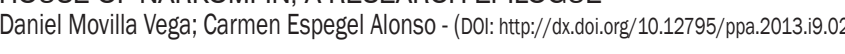

EL SISTEMA COMO LUGAR. TRES ESTRATEGIAS DE COLECTIVIZACIÓN DEL ESPACIO

STRATEGIES OF THE CONTEMPORARY DOMESTIC SPACE

LA CASA EN “CAMPO DE ARROZ”. UN IDEOGRAMA DE INTERACCIÓN EN EL HÁBITAT

LA CASA EN "CAMPO DE ARROZ". UN IDEOGRAMA DE INTERACCIÓN EN EL HÁBITAT
JAPONES CONTEMPORÁNEO / HOUSE IN A "RICE PADDY". AN IDEOGRAM OF INTERACTION IN THE CONTEMPORARY JAPANESE HABITAT

LA CONSTRUCCIÓN TEORICA Y PRÁCTICA DE UN NUEVO HÁBITAT MODERNO: UNOS PATIOS Y UNA CALLE (1946-1954) / THE THEORETICAL AND PRACTICAL CONSTRUCTION OF A NEW MODERN

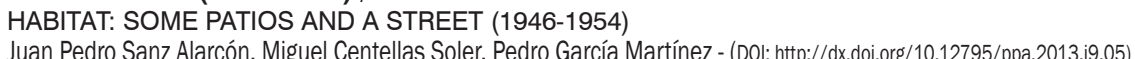

EL BARRIO DE SÃO VICTOR DE ÁLVARO SIZA: ENTRE LA TEORÍA Y LA PRÁCTICA DE LAS OPERACIONES SAAL / SÃO VICTOR DE ÁLVARO SIZA DISTRICT: BETWEEN THEORY AND PRACTICE OF

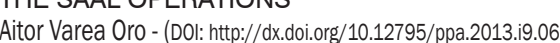

REVISITING PUBLIC SPACE IN POST-WAR SOCIAL HOUSING IN GREAT BRITAIN/ REPENSANDO EL ESPACIO PUBLICO DE LAS VIVIENDAS SOCIALES DE POST-GUERRA EN GRAN BRETAÑA

INTIMIDADES TRANSGREDIDAS: HABITAR EN TRÁNSITO / TRANSGRESSED PRIVACIES: TO LIVE IN

TRANSIT

eseña bibliográfica TEXTOS VIVOS

R.D. MARTIENSSEN LA IDEA DEL ESPACIO EN LA AROUITECTURA GRIEGA

Juan Luis Trillo de Leyva 


\section{PULSANDO LA CUESTIÓN SOBRE LA VIVIENDA Y EL HÁBITAT}

\section{PRESSING THE HOUSING AND HABITAT QUESTION}

Rosa María Añón Abajas

RESUMEN En la actualidad es reconocible una alarmante situación en lo relativo al problema de la vivienda. No solo en el mundo subdesarnolado, sino tambien en los contexios que años atras hablian alcanzado un estado de bienestar bien definido, proliferan situaciones de infravivienda, comos si hubiesen quedado borrados todos los años empeñados en alcanzarlo. En estas circunstancias, da y el hábitat. Cuestiones como los hábitats marginales, la inclusión de la participación ciudadana en los procesos de producción del hábitat, ol a revisión de los valores vigentes de los logros pasados inexplicablemente olvidados, motivaron el lanzamiento de una convocatoria que ha logrado una alta participación. Desde esta editorial se presentan los artículos seleccionados para cristalizar el novenon numero, valorando las aportaciones fundamentales que cada uno ofrece y justrificando su puesto en el orden resultante. SUMMARY oday an alarming situation can be observed, regarding the housing problem. Substandard housing conditions now
proliferate, not only in the developing world, but also where a well-defined state of well-being had been reached years ago. It is as if, every year that had been spent achieving that state has been erased. Under these circumstances, the Editorial Board of "proyecto, progreso, arquitectura" have considered it necessary to dedicate an issue to press home the housing and habitat question. Margiinexplicably, for participation. The selected articles are presented in this editorial to crystallize the ninth issue, assessing the fundamental contributions that each one offers, justifying its place in the resulting order.

KEY WORDS habitat, living; housing; city; sociology

Persona de contacto / Corresponding author : rabajas@us.es. Escuela Técnica Superior de Arquitectura. Universidad de Sevilla on el lema "hábitat y habitar", hace un año retábamos a reflexionar sobre esta cuestión que acumula tantos problemas no resueltos por los procesos establecidos de producción. suficientemente la gravedad del problema.

Los abusos del desarrollismo desencadenaron críticas a la tecnocracia y consecuentes intentos de superación del Movimiento Moderno, pero en su lugar no siempre hubo alternativas válidas. Aquellas críticas fueron aprovechadas para tumbar ideales utópicos y entronar un nefasto pragmatismo que sólo ha contribuido a agudizar el problema. Aunque Gueddes demostrase que la diversidad cultural es una incontestable realidad, que los hábitats no admiten ser extrapolados $y$, consecuentemente, sea imposible creer en ningún modelo internacional, aún cabe pensar que algunos fracasos . Una alarmante realidad, obliga a afrontar urgente y comprometidamente el reto de investigar $y$ ensayar propuestas para ofrecer soluciones, ho basta denunciar o deschbir los casos. Sin embargo no resulla casual que aly os conocidos discusos

Desde el Consejo Editorial se sugirieron líneas de trabajo que pretendían incluir todas las contribucióces posibles, conflando en que las investigaciones mejor defendidas, acabasen cristalizando el taciones de los investigadores en materia de habitar La masorí incluesentan las pincipales aporla cuestión sociológica y la preos mán por considerar al habitante en el proceso de producción del hábitat La cuestión sobre la marginalidad presente en todos de modo indirecto, se aborda más directamente en los dos atrulos finates del número. Sten Gromark en su texto La primací de la calidad

Calidad residencial en la creación urbana. Una observacion a a importancia de la producción de Henri Raymond

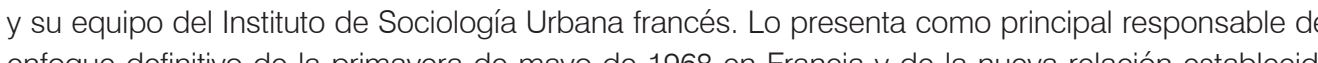
desde entonces entre sociologí y arquitectura Reconociendo la figura recursora de Heni Lefebre - director de la tesis doctorat de Raymond-y citando a los demás investigadores que colaboraon en 
cada uno de los trabajos sucesivos que se fueron encadenando, muestra esta importante linea de investigación que abordó la crítica a la cuestión de la vivienda y el hábitat desde la sociología, ensayando metodos analiticos para comprender la valoración que el habitante hace del espacio construdo, de sus relaciones de vecindad, de su satisfacción con todo ello. Muy importante también es cómo defiende la utilidad de todo ese conocimiento acumulado y sus conclusiones, mencionando una serie de reconocidas obras de reciente construccion, presentadas como consecuencia. Este texto que reclama la investigación sistemática para mejorar la calidad de la vivienda y la ciudad, resulta por todo ejemplar y didáctico, además de oportuno. Tras su lectura, quedamos en una adecuada disposición anímica para continuar con los siguientes.

El artículo Hacia la nueva sociedad comunista: la casa de transición del Narkonfin, epilogo sobre una investigación, expone con rigor y claridad este ensayo ejemplar de modelo residencial, relacionando antecedentes, planteamientos, definición de tipos, materialización y utilización, llegando hasta una evaluación final tras la realización del experimento y la publicación de las conclusiones correspondientes. La narración de la historia es detallada y a su vez sintetica, sirvienndose de extraordinarios dibujos elaborados expresamente para este estudio, con los que muestra la complejidad de los trabajos de indagación tipológica que a lo largo de la segunda década del siglo XX apoyaron la experiencia.

En tercer lugar, El sistema como lugar. Tres estrategias de colectivización del espacio doméstico contemporáneo, repasa teorías fundamentales sobre la habitación; cotejándolas con determinadas obras residenciales que hoy concentran la atención de todos, evidencia el empleo de ciertas estrategias de parametrización de la forma, en base a los tres conceptos que constituyen la base teórica del espacio de habilación colectiva contemporánea -torma, limite y liempo-. Ideas florecidas en el pasado siglo XX, que siguen vivas y activas en la arquitectura residencial que actualmente triunfa. La optimización dimensional de Kein se da en Gifu ejemplo que representa la actual inquietud por la innovación. El sistema jerarquizado dervado de los trabajos de Chemayef Alexander se da en las conocidas propuestas de Druot, Lacatony las teorías destiladsas por Habraken tienen reflejo on lo

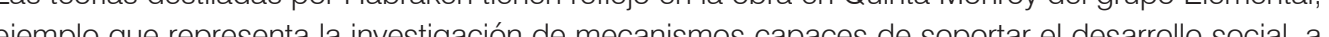

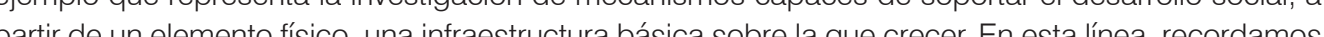
partir de un los magistrales como a caso de Quinta de Mala que cra évora obra liderada por Átvas Siza y que ya cuenta con años de vida. El ejemplo chileno resulta en esta ocasín más oportuno por Siza y que ya co de la clase dirigente por implicarse decididamente en solucionar el problema de la vivienda.
El artículo La casa en "campo de arroz". Un ideograma de interacción en el hábitat japonés contemporáneo, an liza la evolucion del concepto tradicional de la casa unifiamilar japonesa a modelos actuales que manteniendo los elementos permanentes y fuertemente arraigados, superponen otros nuevos, en correspondencla a nuevos hábilos sociales que priman las relaciones con el espacio exterior. La casa unifamiliar, convertida en laboratorio de ideas, aplica tradición y nuevas teorias, registrando las mutaciones de las nuevas forma de habitar. La interacción con los articulos precedentes, revela ciertas conthildades concepluales entre la vivenda unifamiliar y la colectiva.

La construcción teorica y practica de un nuevo habitat urbano. Unos patios y una calle repasa algunas experiencias residenciales en relación a los sucesivos CIAM, refiriendo como los debates las conclusiones fueron evolucion en re casas patio desarrolladas en la periferia de Casablanca por Ecochard y Candilis, un ejemplo clave para demostrar cómo las teorias de la modernidad pueden reaccionar para su necesaria adecuación a lugares y culturas diferentes

El barrio de São Victor de Álvaro Siza: entre la teoría y la práctica de las Operaciones SAAL, artículo muy consistente, que reivindica este caso como contribución esencial al campo de la intervención sobre la ciudad construida, argumentando lo novedoso de la experiencia en todos los factores que comprende, destacando al habitante como un elemento fundamental para la definición de la propuesta y la gestación teórica que requirió diez anos de investigación previa. Aborda cuestiones como la gestión cooperativa de viviendas, el realojo de los vecinos en su mismo ámbito, los fundamentos culturales de una sociedad, la integración de todas estas realidades en una propuesta consciente Otras por Otras problemáticas del hábilat contemporáneo comienzan a ser observadas con la ambición de

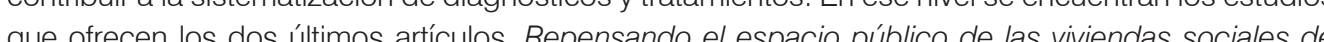
que ofrecen los dos ultimos articulos. Repensando el espacio público de las vivendas sociales de post-guera en Gran Brelana, expone un proceso para el reconocimiento de los factores que proucan la obsolescencla del espacio pú́rco en deterninados ban habitar de indigentes sin techo mediante el estudio de tres casos en la ciudad de Sevilla para lam ha néćn sido la única contribuín que ha entrentado el reto que supone este tema; quien se interese por esta

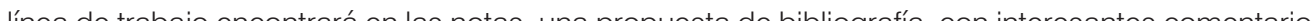

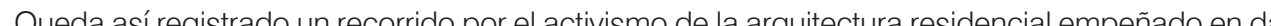
soluciones al problema de la vivienda desde todos los ángulos, evidenciando el recurrente protagonismo de la investigación como fuente de conocimiento aplicable. Esperamos que sirva. в 